\title{
Calcification of peritoneum and peritoneal fluid perfusion malfunction in carcinomatosis of serous membranes of perito- neal cavity
}

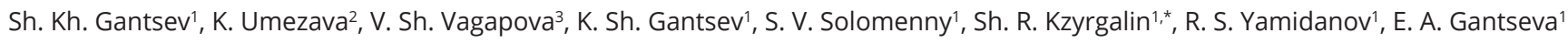 \\ Scientific Research Institute of Oncology, Bashkortostan State Medical University, Lenina street 3, 450000 Ufa, Russian Federation \\ 2 Department of Molecular Target Medicine Screening, School of Medicine, Aichi Medical University, Nagakute, Aichi, 480-1195, Japan \\ ${ }^{3}$ Department of Human anatomy, The Bashkir State Medical University, lenina street 3, 450000, Ufa, Russian Federation
}

\begin{abstract}
The article describes the peritoneal calcification in peritoneal carcinomatosis, as well as its possible role in the development of carcinomatosis within the frames of the authors' alternative theory. The analysis of the "serous-lymph hatches" condition of the intact peritoneum and peritoneum in carcinomatosis was carried out. Also the elemental quantitative calcium determination in the intact peritoneum and the peritoneum in peritoneal carcinomatosis was carried out using the atomic emission spectrometry.
\end{abstract}

Keywords: peritoneum; carcinomatosis; serous-lymphatic hatch; calcification; scanning electron microscopy; spectroscopy

\section{Introduction}

The malfunction of calcium metabolism in the tissues of the body is called calcification (syn. calcareous degeneration). Its morphological manifestation is the loss of calcium from the dissolved state and the deposition and accumulation of calcium salts in cells or in intercellular substance that has degenerated and become necrotic. The array for this process may be the mitochondria and cells' lysosomes, glycosaminoglycans of the basic substance, collagen and elastic fibers. There are some publications that describe the phenomenon of serous membranes calcification [1-3]. The authors of these studies describe the process in specific infectious diseases, mixed dystrophy, systemic autoimmune diseases, cardiovascular diseases, in terms of chronic inflammation. The emergence of this phenomenon indicates a more severe course of the main disease and poor prognosis. The calcification in peritoneal carcinomatosis (PC) of the peritoneum is not described.

In the available literature it is possible to find a description of the manifestations of peritoneal calcification accompanied by fibrosis of all peritoneum layers, its thickening, elasticity and extensibility malfunction without any preceding acute inflammatory diseases of the peritoneum itself $[4,5]$. In this case, the chemical composition of calcium salts in the calcified tissue is qualitatively consistent with the compounds of calcium in the skeletal bones. A synonym to calcification that can often be found is petrification (petrifaction). It is a local calcification, the main reason of which is a profound depletion of tissue that promotes the calcium absorption from the blood and tissue fluid. In this case calcareous accumulations of various sizes and stone density are formed in tissues (the petrificates) around which the reactive inflammation appears $[6,7]$. It is known that in blood calcium has three different forms: nonfiltrable, free ultrafiltrable and ionized. It is the ionized calcium that has a key role in the regulation of physiological processes [8]. It is believed that up to $20-30 \%$ of cancerous tumors are accompanied by hypercalcemia. Pathogenesis and clinical manifestations of paraneoplastic hypercalcaemia depend on a tumor type $[9,4]$. Hypercalcemia occurs most often in lung cancer, breast cancer, kidney cancer, ovarian cancer $[1,2]$.

The detailed study of serous-lymph hatches (SLH) of the abdominal cover that are a part of the lymphatic system and participate in lymphogenous theory of tumor cells dissemination in cancer, allowed us to assume that the

*Corresponding author: Sh. R. Kzyrgalin, Scientific Research Institute of Oncology, Bashkortostan State Medical University, Lenina street 3, 450000 Ufa, Russian Federation. Tel./Fax: +7 34723743 58; Email: kafonco@mail. ru

Received 6 June 2016 Revised 11 August 2016 Accepted 22 August 2016 Published 30 August 2016

Citation: Gantsev SK, Umezava K, Vagapova VS, Gantsev KS, Solomenny SV, Kzyrgalin SR, Yamidanov RS, Gantseva EA. Calcification of peritoneum and peritoneal fluid perfusion malfunction in carcinomatosis of serous membranes of peritoneal cavity. J Cancer Res Ther. 2016; 4(4):38-41. DOI: 10.14312/2052-4994.2016-7

Copyright: (C2016 Gantsev SK, et al. Published by NobleResearch Publishers. This is an open-access article distributed under the terms of the Creative Commons Attribution License, which permits unrestricted use, distribution and reproduction in any medium, provided the original author and source are credited. 
calcification of these structures has a significant impact on the perfusion of the peritoneal fluid in the abdominal cavity [10-14].

At the moment there is no data in the literary sources on the content of calcium in the serous membranes in normal and cancer, as well as participation of SLH in peritoneal calcification. Perhaps in the early stages of cancer, the disorder of calcium metabolism is reversible, and in advanced forms it is manifested by different types of complications in the form of calcium deposits in the tissues and organs that macro-and microscopically reflected in specific tissue damage. The solution to this problem would enable the scientific review of the principles of drug cytoreduction in patients with cancer.

\section{Materials and methods}

The research was carried out at the Research Institute of Oncology of the Bashkir State Medical University in collaboration with the Institute of Metals Superplasticity Problems and the Center of Hygiene and Epidemiology of the Republic of Bashkortostan (Ufa). In order to achieve the objective of the research, the biological materials have been studied. They were collected from suddenly deceased people (the control group) who had no history of cancer (5 dead bodies) and in patients during surgeries (10 cases) for gastric cancer (stage IV) and ovarian cancer (stage IV) with symptoms of PC (clinical group IV). Carcinomatosis nodes in the observations did not exceed $5 \mathrm{~mm}$. Their number varied over a wide range: from 5 to 30. The patients with ovarian and stomach neoplasms were distributed equally ( 5 cases each). In general, the number of females prevailed among the patients ( 6 people). There were no cases of severe obesity in the group of cancer patients and the control group. Also, there were observed no cases of severe heart, liver or kidney diseases, abnormalities of the hemostatic system among these patients and the control group. All people, included in the study, had no serious abdominal surgeries in the past; their age varied from 55 to 60 years.

The areas of parietal peritoneum of the pelvis and diaphragm abdominal muscle sized $5 \times 5 \mathrm{~mm}$ were exsected. In the control group, the peritoneum exsection was performed within the anatomical area (pelvis, diaphragm); at the same time in PC, we preferred the same areas with zones having carcinomatosis nodes. All areas of the peritoneum were deepithelialized before the study.

We used a scanning electron microscope Tescan Vega$3 \mathrm{SBH}$ with tungsten hot cathode as a tool for profound nano-anatomic study. Drying and cleaning the surface of the samples from contamination was carried out with the help of "Fine coat lon sputter JFC 1100". In order to get highresolution pictures on the same equipment, sputtering of the test surface with a thin layer of gold was carried out to discharge and screen the incident beam of accumulated in the volume of material charge. Quantitative determination of the calcium in the test sample was measured by atomic emission spectrometry with microwave plasma generation (Agient 4100 MP-AES), up to five sections of the parietal peritoneum sized $5 \times 5 \mathrm{~mm}$ and having weight of $5-7 \mathrm{~g}$ were studied.

\section{Results and discussion}

Our research of abdominal cover in disseminated ovarian and stomach cancer revealed a number of interesting facts, from a scientific point of view. This blocking of SLH and evident accumulation of salts on the surface of the peritoneum (diaphragmatic and pelvic).

Figure 1 shows the data of SLH scanning electron microscopy of diaphragmatic peritoneum in carcinomatosis located in different functional states. Lumen of most SLH is free from tumor cells, has the form of transverse slots separated by trabeculae: these are functionally valid SLH. There are also SLH defined in different shapes and sizes, totally blocked by tumor cells. This observation is one of the major pathogenetic factors complicating the perfusion of peritoneal fluid, when the imbalance in the system "outflow-inflow" is occurred. Functions of the blocked SLH are taken by the SLH that are still free from tumor masses. Progression of PC leads to occlusion of a growing number of SLH that is clinically reflected by different types of complications, in particular, ascites and interstitial edema. It should be noted that the accumulation of fluid in the interstitial space may happen due to the increased transudation and also as a result of its movement from the intracellular space into the extracellular extravascular space in different types of osmolality changes of biological fluids. It should also be remembered that in this category of patients the processes of malfunction of fluid resorption from the abdominal cavity can also be affected by hemodynamic heart failure and/ or venous stasis in hypertension of the portal vein.

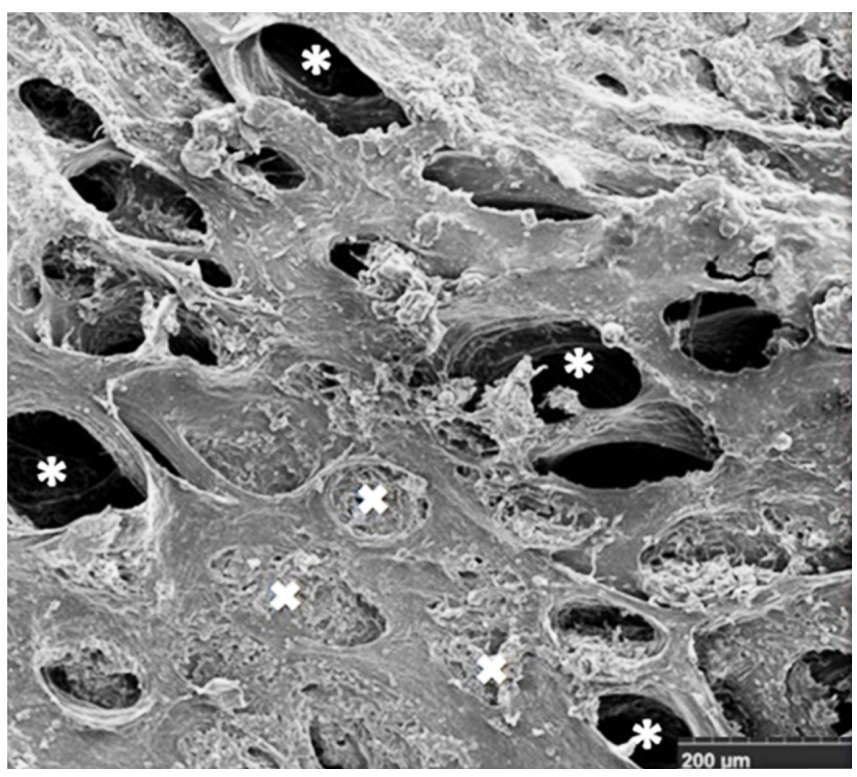

Figure 1 Scanning image of a parietal peritoneum area in carcinomatosis (diaphragmatic region). Functionally valid $(\mathrm{x})$ and blocked by tumor cells $(*)$ serous-lymp hatches are defined.

Figure 2 shows the data of SLH scanning electron microscopy of diaphragmatic peritoneum in norm and in carcinomatosis with symptoms of peritoneal cover calcification. Analyzing the data of SLH scanning images, we come to the preliminary conclusion that patients with malignant tumors in the process of PC development have a calcification of the peritoneum in its various forms (polypoid, coral-like and infiltrative), entailing functional 
malfunction of peritoneum perfusion properties: changes in volume and lymph flow direction that determines the extent of metastasis. At the same time, salt deposits in the lymphatic vessels, SLH are favorable basis for the metastatic cascade development.

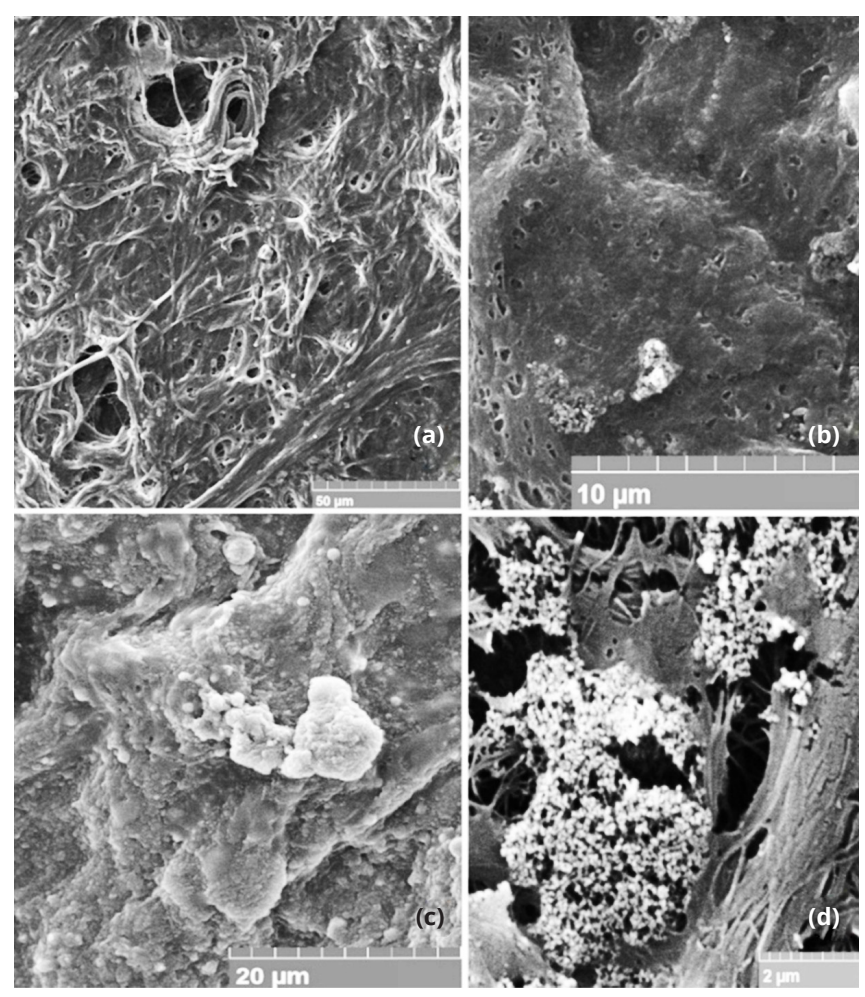

Figure 2 Scanning image of the intact area of the parietal peritoneum (diaphragmatic region) in carcinomatosis: (a) after peeling off mesothelial cover, (b) infiltrative calcification, (c) polypoid calcification, (d) coral-like calcification.

Based on the data obtained, we believe that the polypoid calcification of the peritoneum is the result of total incrustation of one SLH; the coral-like is the results of total incrustation of a few SLH. As far as the infiltrative calcification is concerned, we believe that it is a mixed mechanism of salt formation in peritoneum layers.

The results of atomic emission spectral analysis of intact parietal peritoneum and the peritoneum in PC with the phenomena of SLH calcification are presented in Table 1 and 2. Calcium content in intact diaphragm and pelvic peritoneum was $121.06 \pm 1.21$ and $131.12 \pm 1.36 \mathrm{mg} / \mathrm{kg}$, respectively $(p<0.0001)$. Calcium content in the diaphragm and pelvic peritoneum in PC with the phenomena of calcification was $1205.5 \pm 24.09$ and $1405.48 \pm 12.25 \mathrm{mg} /$ $\mathrm{kg}$, respectively $(\mathrm{p}<0.0001)$. Thus, the calcium content in the diaphragm and the pelvic peritoneum in PC with the phenomena of calcification exceeds the norm 9.96 and 10.72 times respectively.

The data reveals calcium metabolism disorders in this category of patients and latemanifestation of complications, such as salt deposits in the abdominal cover.

The obtained data, including electron microscopy, quantitative determination of calcium, allow us to suggest a particular role of SLH and peritoneum calcification in the development and progression of PC.
Table 1 Comparative indicators of calcium in the intact peritoneum and the peritoneum in carcinomatosis with signs of serous-lymp hatches calcification

\begin{tabular}{ccccc}
\hline & \multicolumn{4}{c}{ Calcium content $(M+m), m g / \mathrm{kg}$} \\
\cline { 2 - 5 } $\begin{array}{c}\text { Sample } \\
\text { number }\end{array}$ & diaphragmatic peritoneum & pelvic peritoneum \\
\cline { 2 - 5 } & $\begin{array}{c}\text { intact } \\
\text { peritoneum }\end{array}$ & $\begin{array}{c}\text { peritoneum } \\
\text { in } P C\end{array}$ & $\begin{array}{c}\text { intact } \\
\text { peritoneum }\end{array}$ & $\begin{array}{c}\text { peritoneum } \\
\text { in } P C\end{array}$ \\
\hline 1 & 122.0 & 1124.1 & 134.9 & 1446.4 \\
2 & 118.3 & 1213.5 & 128.2 & 1403.3 \\
3 & 119.6 & 1273.4 & 130.3 & 1387.8 \\
4 & 125.3 & 1196.2 & 133.7 & 1375.1 \\
5 & 120.1 & 1220.3 & 128.5 & 1414.8 \\
\hline
\end{tabular}

Table 2 The calcium content in the intact peritoneum and the peritoneum in peritoneal carcinomatosis with signs of serous-lymph hatches calcification (diaphragmatic and pelvic area) $(\mathrm{P}<0.0001)$

\begin{tabular}{lcc}
\hline Anatomical area & \multicolumn{2}{c}{$\begin{array}{c}\text { Calcium content } \\
(M+m), m g / k g\end{array}$} \\
\cline { 2 - 3 } & intact peritoneum & peritoneum in $P C$ \\
\hline Diaphragmatic peritoneum & $121.06 \pm 1.21$ & $1205.5 \pm 24.09$ \\
Pelvic peritoneum & $131.12 \pm 1.36$ & $1405.48 \pm 12.25$ \\
\hline
\end{tabular}

This pathogenetic mechanism is consistent with an alternative theory of PC according to which cancer cells transfer from the first order body to the abdominal cover is executed lymphatically [10, 11]. Calcification of the parietal peritoneum in cancer and PC is described by us for the first time. The classification of calcification of the peritoneum and its appendages is presented for the first time, which may be a prognostic factor of PC. We believe that this data will serve as a reference point of the drug therapy optimization for the metastasis preventing and reducing its scope in cancer.

The research in this area is being continued.

\section{Conclusion}

The peritoneum calcification with the development of specific lesions of abdominal cover in the form of salt deposits of various manifestations is observed in cancer of the peritoneal cavity with the PC phenomena. The calcium content in the diaphragmatic and pelvic peritoneum in PC with the calcification phenomena exceeds the norm 9.96 and 10.72 times respectively. Salt occlusion of peritoneum SLH alters the scope of PC.

\section{Acknowledgments}

We express our gratitude and deep appreciation to Shamil K. Gantsev, Head of Research Institute of Oncology of Bashkir State Medical University, MD, Professor, corresponding member of Academy of Sciences of Republic of Bashkortostan, Honored Scientist of the Russian Federation for the valuable advice and comments on the article. 


\section{Conflicts of interest}

Group of authors declares no conflict of interest in determining the structure of the research, the collection, analysis and interpretation of data.

\section{References}

[1] Bespalov I, Some preliminary data on the state of calcium metabolism in patients with stomach cancer. Radiation diagnostics in Oncology Clinic: Collection of scientific papers. 1988; 138-148.

[2] Gantsev Sh, Solomenny S, Ishmuratova R, Kzyrgalin Sh.R, Khalikov LV, et al. Capabilities of atomic force microscopy in nano-anatomic studies (on the example of an intact and carcinomatosis peritoneum). Creative oncology and surgery. 2014; 1.

[3] Gantsev Sh. Peritoneal carcinomatosis - new look at the problem. Creative oncology and surgery 2013; 1.

[4] Gantsev Sh. Presentations: Peritoneal carcinomatosis- a new look at the problem. Creative oncology and surgery. 2013; 1.

[5] Gantsev Sh, Solomenny S, Ishmuratova R, Kzyrgalin Sh.R, Khalikov LV, et al. Application of high-resolution scanning electron microscopy in nano-anatomic studies of peritoneum. Creative oncology and surgery. $2013 ; 1$.

[6] Ishmuratova R, Kzyrgalin Sh, Gantsev K, et al. Anatomical prerequisites for the development of peritoneal carcinomatosis.Literature analysis and own data. Creative surgery and oncology. 2013; 3:74-79.

[7] Berridge $M$, Bootman $M$, Roderick $H$. Calcium signalling: dynamics, homeostasis and remodeling. Nat Rev Mol Cell Biol. 2003; 4(1):517529.

[8] Di Paolo N, Sacchi G. Atlas of peritoneal histology. Perit Dial Int. 2000; 20 Suppl 3:S5-81.

[9] Di Paolo N, Garosi G. Peritoneal sclerosis. J Nephrol. 1999; 12(6):347361.

[10] Di Paolo N, Sacchi G, Lorenzoni P, Sansoni E, Gaggiotti E. Ossification of the peritonel membrane. Perit Dial Int. 2004; 24(5):471-477.

[11] Itano N, Hatano S. F-Actin bundling protein from Physarum polycephalum: Purification and its capacity for co-bundling of actin filaments and microtubules. Cell Motil Cytoskel. 1991; 19(4):244-254.

[12] Klemm G. Peritoneal calcification and calciphylaxis. Nephron. 1989; 51(1):124.

[13] Monteith GR, McAndrew D, Faddy HM, Roberts-Thomson S]. Calcium and cancer: targeting Ca2+ transport. Nat Rev Cancer. 2007; 7(7):519530.

[14] Prevarskaya N, Skryma R, Shuba Y. Calcium in tumor metastasis: new roles for known actors. Nat Rev Cancer. 2011; 11(8):609-618. 